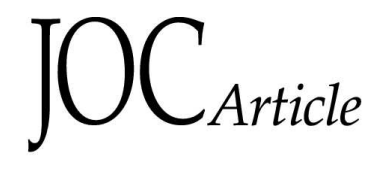

\title{
A Fine-Tuned Molybdenum Hexacarbonyl/Phenol Initiator for Alkyne Metathesis
}

\author{
Supplementary Information
}

Volodymyr Sashuk, Jolanta Ignatowska and Karol Grela*

Institute of Organic Chemistry, Polish Academy of Sciences, Kasprzaka 44/52, 01-224

Warsaw, Poland

grela@icho.edu.pl

\section{Contents}

1. General ................................ 2

2. General Procedure for Ring Closing Alkyne Metathesis and Alkyne Cross Metathesis . . . . . . 2

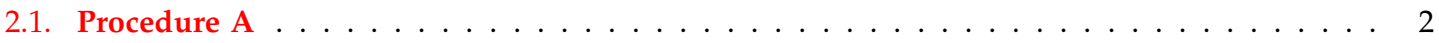

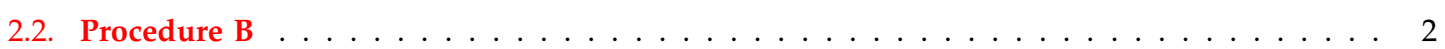

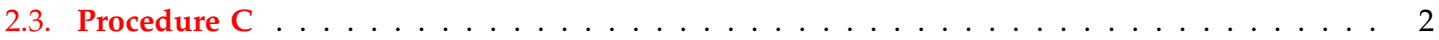

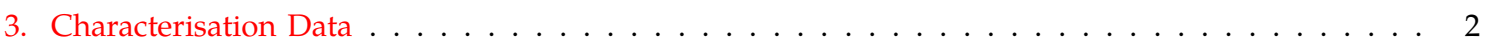

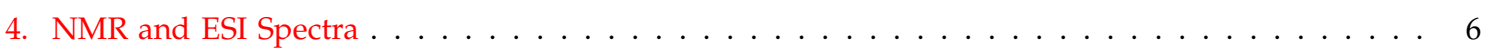




\section{General}

Unless otherwise noted, all reactions were carried out under Ar in pre-dried glassware using Schlenk techniques. The solvents were dried by distillation over the following drying agents and were transferred under argon: THF (K/benzophenone), toluene ( $\mathrm{Na}), n$-pentane, $n$-hexane, $\mathrm{CH}_{2} \mathrm{Cl}_{2}\left(\mathrm{CaH}_{2}\right), \mathrm{Et}_{2} \mathrm{O}\left(\mathrm{LiAlH}_{4}\right), \mathrm{MeOH}(\mathrm{Mg})$. Flash column chromatography: Merck silica gel 60 (230-400 mesh). NMR: Spectra were recorded in $\mathrm{CDCl}_{3}$; chemical shifts $(\delta)$ are given in ppm relative to TMS, coupling constants $(J)$ in Hz. Micro-analyses were provided by Institute of Organic Chemistry, PAS, Warsaw. All commercially available chemicals were used as received.

\section{General Procedure for Ring Closing Alkyne Metathesis and Alkyne Cross Metathesis}

\subsection{Procedure A}

A solution of diyne 2 or alkyne(s) $4(1 \mathrm{mmol}), \mathrm{Mo}(\mathrm{CO})_{6}(13-26 \mathrm{mg}, 0.05-0.1 \mathrm{mmol}, 5-10 \mathrm{~mol} \%)$ and 2-fluorophenol $(112 \mathrm{mg}, 1 \mathrm{mmol})$ in chlorobenzene $(20 \mathrm{~mL})$ was refluxed for $0.25-6 \mathrm{~h}$ using electric heating mantle. After evaporation of the solvent, the residue was purified by flash chromatography on silica gel (c-hexane/ethyl acetate 4:1-99:1) to give products 3 or 5 or $\mathbf{6}$.

\subsection{Procedure B}

A solution of diyne 2 or alkyne $(\mathrm{s}) 4(1 \mathrm{mmol}), 3$-hexyne $(8 \mathrm{mg}, 0.1 \mathrm{mmol}), \mathrm{Mo}(\mathrm{CO})_{6}(13-26$ $\mathrm{mg}, 0.05-0.1 \mathrm{mmol}, 5-10 \mathrm{~mol} \%)$ and 2-fluorophenol (112 mg, $1 \mathrm{mmol})$ in chlorobenzene (20 $\mathrm{ml}$ ) was refluxed for $0.5-4 \mathrm{~h}$ using electric heating mantle. After evaporation of the solvent, the residue was purified by flash chromatography on silica gel (c-hexane/ethyl acetate 4:1-99:1) to give products 3 or 5 or 6 .

\subsection{Procedure C}

A solution of diyne 2 or alkyne(s) $4(1 \mathrm{mmol})$, 3-hexyne $(8 \mathrm{mg}, 0.1 \mathrm{mmol}), 1,2$-diphenoxyethane (24 mg, $0.11 \mathrm{mmol}), \mathrm{Mo}(\mathrm{CO})_{6}(26 \mathrm{mg}, 0.1 \mathrm{mmol}, 10 \mathrm{~mol} \%)$ and 2-fluorophenol (112 mg, 1 $\mathrm{mmol})$ in 1,2-dichloroethane $(15 \mathrm{ml})$ was refluxed for 1-20 h. After evaporation of the solvent, the residue was purified by flash chromatography on silica gel (c-hexane/ethyl acetate 4:1-99:1) to give products products 3 or $\mathbf{5}$ or $\mathbf{6}$.

\section{Characterisation Data}

1,6-Dioxacyclodec-9-yne-2,5-dione (3a). Yellow solid. ${ }^{1} \mathrm{H}$ NMR (500 MHz, $\left.\mathrm{CDCl}_{3}\right) \quad \delta 2.47$ (m, $4 \mathrm{H}), 2.70(\mathrm{~s}, 4 \mathrm{H}), 4.27(\mathrm{t}, J=5.7 \mathrm{~Hz}, 4 \mathrm{H}) ;{ }^{13} \mathrm{C} \mathrm{NMR}\left(125 \mathrm{MHz}, \mathrm{CDCl}_{3}\right) \quad \delta 19.6(\mathrm{~s}), 29.9(\mathrm{~s}), 61.3$ 
(s), 78.7 (s), 171.7 (s); EI-MS m/z (rel intensity) $196\left(\mathrm{M}^{+\bullet}, 1\right), 166$ (1), 101 (20), 78 (100), 66 (75), 55 (20), 39 (35). Analytical data are in agreement with those published in literature. ${ }^{1}$

1,12-Bis[(4-methylphenyl)sulfonyl]-1,12-diaza-6,17-cyclodocosadiyne (3c). Colorless crystals. IR (film) $\nu$ 2938, 2866, 1599, 1459, 1339, 1157, 1090, 972, 814, 752, 655, $549 \mathrm{~cm}^{-1} ;{ }^{1} \mathrm{H}$ NMR $\left(500 \mathrm{MHz}, \mathrm{CDCl}_{3}\right) \quad \delta 1.35-1.40(\mathrm{~m}, 8 \mathrm{H}), 1.55-1.60(\mathrm{~m}, 8 \mathrm{H}), 2.05-2.10(\mathrm{~m}, 8 \mathrm{H}), 2.33(\mathrm{~s}, 6 \mathrm{H})$, 2.99-3.05 (m, 8H), 7.16-7.22 (m, 4H), 7.55-7.60 (m, 4H); ${ }^{13} \mathrm{C}$ NMR $\left(125 \mathrm{MHz}, \mathrm{CDCl}_{3}\right) \quad \delta 18.2$ (s), $21.5(\mathrm{~s}), 25.8(\mathrm{~s}), 27.4(\mathrm{~s}), 47.7(\mathrm{~s}), 80.2(\mathrm{~s}), 127.1$ (s), $129.6(\mathrm{~s}), 136.8(\mathrm{~s}), 143.0(\mathrm{~s})$; ESI calcd for $\mathrm{C}_{34} \mathrm{H}_{46} \mathrm{~N}_{2} \mathrm{O}_{4} \mathrm{~S}_{2} \mathrm{Na}$ 633.2791, found 633.2818.

1,8-Dioxacyclotetradec-11-yne-2,7-dione (3d). White solid. ${ }^{1} \mathrm{H}$ NMR (500 MHz, $\left.\mathrm{CDCl}_{3}\right) \quad \delta$ $1.73-1.78(\mathrm{~m}, 4 \mathrm{H}), 2.37-2.41(\mathrm{~m}, 4 \mathrm{H}), 2.52(\mathrm{t}, J=5.6 \mathrm{~Hz}, 4 \mathrm{H}), 4.14(\mathrm{t}, J=5.5 \mathrm{~Hz}, 4 \mathrm{H}) ;{ }^{13} \mathrm{C}$ NMR (125 MHz, $\left.\mathrm{CDCl}_{3}\right) \quad \delta 19.0$ (s), 24.9 (s), 34.9 (s), 62.4 (s), 77.8 (s), 173.1 (s); EI-MS m/z (rel intensity) $224\left(\mathrm{M}^{+\bullet}, 1\right), 101(10), 78(100), 55$ (50), 39 (50). Analytical data are in agreement with those published in literature. ${ }^{1}$

$\mathbf{N}^{1}$-(2,5-dioxo-1,6-dioxa-9-cyclododecynyl)-2,2,2-trifluoroacetamide (3e). Colorless oil. IR (film) $\nu$ 3423, 3332, 2968, 1753, 1734, 1543, 1391, 1290, 1210, 1175, 1046, $1006 \mathrm{~cm}^{-1}$; ${ }^{1}$ H NMR (500 MHz, $\left.\mathrm{CDCl}_{3}\right) \quad \delta 2.33-2.40(\mathrm{~m}, 1 \mathrm{H}), 2.47-2.65(\mathrm{~m}, 3 \mathrm{H}), 2.92(\mathrm{dd}, J=17.8$ and $3.6 \mathrm{~Hz}, 1 \mathrm{H}), 3.26(\mathrm{dd}$, $J=17.8$ and $4.2 \mathrm{~Hz}, 1 \mathrm{H}), 3.86-3.92(\mathrm{~m}, 1 \mathrm{H}), 4.08-4.15(\mathrm{~m}, 1 \mathrm{H}), 4.36-4.42(\mathrm{~m}, 1 \mathrm{H}), 4.87-4.95(\mathrm{~m}$, 2H), 7.55 (bs, 1H); ${ }^{13} \mathrm{C}$ NMR (125 MHz, $\left.\mathrm{CDCl}_{3}\right) \quad \delta 18.9$ (s), 20.2 (s), 35.9 (s), 48.9 (s), 61.4 (s), 64.0 (s), 78.3 (s), 78.6 (s), 115.6 (q), 156.9 (q), 168.8 (s), 170.9(s); ESI calcd for $\mathrm{C}_{12} \mathrm{H}_{12} \mathrm{~F}_{3} \mathrm{NO}_{5} \mathrm{Na}$ 330.0560 , found 330.0550 .

1-(2-Phenyl-1-ethynyl)benzene (5a). White solid. IR (KBr) $\nu$ 1492, 1441, 756, 689, $508 \mathrm{~cm}^{-1}$; ${ }^{1} \mathrm{H}$ NMR $\left(400 \mathrm{MHz}, \mathrm{CDCl}_{3}\right) \quad \delta$ 7.31-7.38 (m, 6H), 7.51-7.56 (m, 4H); ${ }^{13} \mathrm{C}$ NMR (100 MHz, $\left.\mathrm{CDCl}_{3}\right) \quad \delta 89.3$ (s), $123.3(\mathrm{~s}), 128.2$ (s), 128.3 (s), 131.6 (s); EI-MS m/z (rel intensity) 179 (15), 178 $\left(\mathrm{M}^{+} \bullet, 100\right), 177$ (12), 176 (20), 76 (10); HRMS calcd for $\mathrm{C}_{14} \mathrm{H}_{10}$ 178.0782, found 178.0782; Anal calcd for $\mathrm{C}_{14} \mathrm{H}_{10}$ C, 94.34; $\mathrm{H}, 5.66$; Found $\mathrm{C}, 94.07 ; \mathrm{H}, 5.67$.

1-(Trifluoromethyl)-3-2-[3-(trifluoromethyl)phenyl]-1-ethynylbenzene (5b). White solid. IR (film with $\mathrm{CH}_{2} \mathrm{Cl}_{2}$ ) $\nu$ 3075, 2925, 1589, 1494, 1440, 1347, 1329, 1289, 1263, 1168, 1129, 1072, 801, $695 \mathrm{~cm}^{-1} ;{ }^{1} \mathrm{H}$ NMR $\left(400 \mathrm{MHz}, \mathrm{CDCl}_{3}\right) \quad \delta$ 7.48-7.54 (m, 2H), 7.60-7.64 (m, 2H), 7.69-7.74 (m, 2H), 7.80-7.83 (m, 2H); ${ }^{13} \mathrm{C}$ NMR (100 MHz, $\left.\mathrm{CDCl}_{3}\right) \quad \delta 89.2(\mathrm{~s}), 123.5$ (s), $123.6(\mathrm{q}), 125.3(\mathrm{q})$, 128.5 (q), 129.0 (s), 131.2 (q), 134.7 (s); EI-MS m/z (rel intensity) 315 (18), 314 (M+•, 100), 295 (12), 245 (4), 225 (4); HRMS calcd for $\mathrm{C}_{16} \mathrm{H}_{8} \mathrm{~F}_{6}$ 314.0530, found 314.0516. Analytical data are in

\footnotetext{
1 Fürstner, A.; Guth, O.; Rumbo, A.; Seidel, G. J. Am. Chem. Soc. 1999, 121, 11108-11113.
} 
agreement with those published in literature. ${ }^{2}$

1-Methoxy-2-[2-(2-methoxyphenyl)-1-ethynyl]benzene (5c). White solid. ${ }^{1} \mathrm{H}$ NMR (200 MHz, $\left.\mathrm{CDCl}_{3}\right) \quad \delta 3.93(\mathrm{~s}, 6 \mathrm{H}), 6.85-7.12(\mathrm{~m}, 4 \mathrm{H}), 7.22-7.38(\mathrm{~m}, 2 \mathrm{H}), 7.52(\mathrm{dd}, J=7.6$ and $1.8 \mathrm{~Hz}, 2 \mathrm{H})$; ${ }^{13} \mathrm{C}$ NMR (50 MHz, $\left.\mathrm{CDCl}_{3}\right) \quad \delta 55.9$ (s), 89.7 (s), 110.7 (s), 112.5 (s), 120.4 (s), 129.6 (s), 133.6 (s), 160.2 (s); EI-MS m/z (rel intensity) $238\left(\mathrm{M}^{+\bullet}, 100\right), 223$ (31), 207 (15), 205 (13), 195 (9), 178 (16), 167 (12), 165 (35), 152 (27), 131 (43). Analytical data are in agreement with those published in literature. ${ }^{3}$

1-Ethyl-4-[2-(4-ethylphenyl)-1-ethynyl]benzene (5d). White solid. IR (film with $\left.\mathrm{CH}_{2} \mathrm{Cl}_{2}\right) \quad \nu$ 2968, 2931, 1461, 833, $550 \mathrm{~cm}^{-1}$; ${ }^{1} \mathrm{H}$ NMR $\left(400 \mathrm{MHz}, \mathrm{CDCl}_{3}\right) \quad \delta 1.23(\mathrm{t}, J=7.6 \mathrm{~Hz}, 6 \mathrm{H}), 2.65$ $(\mathrm{q}, J=7.6 \mathrm{~Hz}, 4 \mathrm{H}), 7.15-7.19(\mathrm{~m}, 4 \mathrm{H}), 7.44(\mathrm{dt}, J=8.4$ and $2.0 \mathrm{~Hz}, 4 \mathrm{H}) ;{ }^{13} \mathrm{C} \mathrm{NMR}(100 \mathrm{MHz}$, $\left.\mathrm{CDCl}_{3}\right) \quad \delta 15.4(\mathrm{~s}), 28.8(\mathrm{~s}), 88.9$ (s), 120.6 (s), 127.9 (s), 131.5 (s), 144.5 (s); EI-MS m/z (rel intensity) 235 (16), $234\left(\mathrm{M}^{+} \bullet, 85\right), 220$ (19), 219 (100), 204 (30), 202 (15), 102 (16); HRMS calcd for $\mathrm{C}_{18} \mathrm{H}_{18}$ 234.1408, found 234.1405; Anal calcd for $\mathrm{C}_{18} \mathrm{H}_{18}$ C, 92.26; H, 7.74; Found C, 92.48; $\mathrm{H}, 7.82$. Analytical data are in agreement with those published in literature. ${ }^{4}$

1,10-Bis(tertbutyldimethylsilyloxy)-5-decyne (5e). Colorless oil. IR (film) $\quad \nu$ 2929, 2857, $1471,1462,1387,1361,1253,1101,1005,970,938,832,772,711,660 \mathrm{~cm}^{-1} ;{ }^{1} \mathrm{H}$ NMR (300 MHz, $\left.\mathrm{CDCl}_{3}\right) \quad \delta 0.03(\mathrm{~s}, 12 \mathrm{H}), 0.88(\mathrm{~s}, 18 \mathrm{H}), 1.43-1.63(\mathrm{~m}, 8 \mathrm{H}), 2.15(\mathrm{t}, J=7.0 \mathrm{~Hz}, 4 \mathrm{H}), 3.60(\mathrm{t}, J=6.2$ $\mathrm{Hz}, 4 \mathrm{H}) ;{ }^{13} \mathrm{C}$ NMR $\left(75 \mathrm{MHz}, \mathrm{CDCl}_{3}\right) \quad \delta-4.9$ (s), 18.7 (s), 18.9 (s), 25.9 (s), 26.3 (s), 32.4 (s), 63.2 (s), 80.6 (s); EI-MS m/z (rel intensity) 398 (M+•, 0.7), 383 (2), 341 (12), 209 (26), 147 (10), 135 (72), 107 (19), 93 (44), 75 (86), 73 (65), 67 (40). Analytical data are in agreement with those published in literature. ${ }^{5}$

Methyl 2-(8-[2-(methoxycarbonyl)phenoxy]-4-octynyloxy)benzoate (5f). White solid. IR (KBr) $\nu$ 2947, 1722, 1599, 1492, 1448, 1238, 1172, 1084, $758 \mathrm{~cm}^{-1}$; ${ }^{1} \mathrm{H}$ NMR $\left(400 \mathrm{MHz}, \mathrm{CDCl}_{3}\right) \quad \delta 1.98$ (quint., $J=6.0 \mathrm{~Hz}, 4 \mathrm{H}), 2.41(\mathrm{t}, J=7.0 \mathrm{~Hz}, 4 \mathrm{H}), 3.86(\mathrm{~s}, 6 \mathrm{H}), 4.10(\mathrm{t}, J=6.0 \mathrm{~Hz}, 4 \mathrm{H}), 6.93-6.98$ $(\mathrm{m}, 4 \mathrm{H}), 7.39-7.44(\mathrm{~m}, 2 \mathrm{H}), 7.77(\mathrm{dd}, J=6.0$ and $2.0 \mathrm{~Hz}, 2 \mathrm{H}) ;{ }^{13} \mathrm{C} \mathrm{NMR}\left(100 \mathrm{MHz}, \mathrm{CDCl}_{3}\right) \quad \delta$ $15.3(\mathrm{~s}), 28.6$ (s), 51.9 (s), 67.2 (s), 79.7 (s), 113.1 (s), 120.1 (s), 120.3 (s), $131.6(\mathrm{~s}), 133.4(\mathrm{~s}), 158.5$ (s), 166.8 (s); EI-MS m/z (rel intensity) $410\left(\mathrm{M}^{+\bullet}, 6\right), 227$ (100), 226 (36), 211 (13), 200 (21), 199 (54), 198 (23), 197 (27), 185 (20), 171 (12), 165 (16), 153 (12), 152 (38), 121 (49), 120 (53), 107 (31), 106 (75), 105 (19), 92 (19), 91 (43), 79 (28), 77 (19); HRMS calcd for $\mathrm{C}_{24} \mathrm{H}_{26} \mathrm{O}_{6} 410.1729$, found 410.1728.

1,10-Dibromodec-4-yne (5g). Colorless oil. IR (film) v 2930, 2860, 2230, 1451, 1434, 1333,

\footnotetext{
2 Pschirer, N. G., Bunz U. H.F. Tetrahedron Lett. 1999, 40, 2481-2484.

3 Furstner, A.; Mathes, C. Org. Lett. 2001, 3, 221-223.

4 Han, G. Y.; Han, P. F.; Perkins, J.; McBay, H. C. J. Org. Chem. 1981, 46, 4695-4700.

5 Y. Torisawa et al. Tetrahedron 1991, 38, 8067-8078.
} 
1290, 1250, 646, $562 \mathrm{~cm}^{-1} ;{ }^{1} \mathrm{H}$ NMR $\left(200 \mathrm{MHz}, \mathrm{CDCl}_{3}\right) \quad \delta 1.54-1.72(\mathrm{~m}, 4 \mathrm{H}), 1.88-2.04(\mathrm{~m}, 4 \mathrm{H})$, 2.14-2.26 (m, 4H), $3.43(\mathrm{t}, J=6.7 \mathrm{~Hz}, 4 \mathrm{H}) ;{ }^{13} \mathrm{C} \mathrm{NMR}\left(50 \mathrm{MHz}, \mathrm{CDCl}_{3}\right) \quad \delta 17.8(\mathrm{~s}), 27.3(\mathrm{~s}), 31.7$ (s), 33.2 (s), 79.9 (s); EI-MS m/z (rel intensity) 217 (6), 215 (6), 175 (11), 173 (11), 135 (30), 95 (35), 93 (29), 81 (97), 79 (38), 67 (100), 55 (20), 41 (36). HRMS calcd for $\mathrm{C}_{10} \mathrm{H}_{16}{ }^{79} \mathrm{Br} 215.0435$, found 215.0427.

1-(4-Ethylphenyl)-6-(tertbutyldimethylsilyloxy)-1-hexyne (6a). Colorless oil. IR $\nu$ 2929, 2857, 1510, 1471, 1462, 1387, 1360, 1253, 1104, 1006, 972, 938, 830, 773, 714, $661 \mathrm{~cm}^{-1}$; ${ }^{1} \mathrm{H}$ NMR (300 $\left.\mathrm{MHz}, \mathrm{CDCl}_{3}\right) \quad \delta 0.03(\mathrm{~s}, 6 \mathrm{H}), 0.87(\mathrm{~s}, 9 \mathrm{H}), 1.19(\mathrm{t}, J=7.6 \mathrm{~Hz}, 3 \mathrm{H}), 1.58-1.68(\mathrm{~m}, 4 \mathrm{H}), 2.39(\mathrm{t}, J$ $=6.6 \mathrm{~Hz}, 2 \mathrm{H}), 2.59(\mathrm{q}, J=7.6 \mathrm{~Hz}, 2 \mathrm{H}), 3.63(\mathrm{t}, J=6.0 \mathrm{~Hz}, 2 \mathrm{H}), 7.07(\mathrm{~d}, J=8.2 \mathrm{~Hz}, 2 \mathrm{H}), 7.28(\mathrm{~d}$, $J=8.2 \mathrm{~Hz}, 2 \mathrm{H}) ;{ }^{13} \mathrm{C}$ NMR $\left(75 \mathrm{MHz}, \mathrm{CDCl}_{3}\right) \quad \delta-4.9$ (s), 15.8 (s), 18.8 (s), 19.6 (s), 25.7 (s), 26.4 (s), 29.1 (s), $32.4(\mathrm{~s}), 63.1$ (s), 81.2 (s), 89.7 (s), 121.6 (s), 128.1 (s), 131.9 (s), 144.1 (s); EI-MS m/z (rel intensity) $316\left(\mathrm{M}^{+\bullet}, 0.3\right), 301$ (0.8), 259 (80), 185 (100), 171 (25), 157 (11), 143 (17), 129 (16),75 (80); CI calcd for $\mathrm{C}_{20} \mathrm{H}_{33} \mathrm{SiO} 317.2301$, found 317.2302. Anal calcd for $\mathrm{C}_{20} \mathrm{H}_{32} \mathrm{SiO} \mathrm{C}, 75.88 ; \mathrm{H}$, 10.19; Found C, 75.95; H, 10.18.

1-(1-Butynyl)benzene (6c). Colorless oil. IR (film) $\nu$ 2976, 1600, 1491, 755, $691 \mathrm{~cm}^{-1} ;{ }^{1} \mathrm{H}$ NMR $\left(400 \mathrm{MHz}, \mathrm{CDCl}_{3}\right) \delta 1.24(\mathrm{t}, J=7.0 \mathrm{~Hz}, 3 \mathrm{H}), 2.42(\mathrm{q}, J=8.0 \mathrm{~Hz}, 2 \mathrm{H}), 7.25-7.30(\mathrm{~m}, 3 \mathrm{H}), 7.33-7.41$ $(\mathrm{m}, 2 \mathrm{H}) ;{ }^{13} \mathrm{C}$ NMR (100 MHz, $\left.\mathrm{CDCl}_{3}\right) \quad \delta 13.1$ (s), 13.9 (s), 79.8 (s), 91.6 (s), $124.0(\mathrm{~s}), 127.5$ (s), 128.2 (s), 131.5 (s); EI-MS m/z (rel intensity) $130\left(\mathrm{M}^{+\bullet}, 87\right), 129$ (69), 128 (37), 127 (16), 116 (14), 115 (100); HRMS calcd for $\mathrm{C}_{10} \mathrm{H}_{10} 130.0782$, found $130.0782 .{ }^{6}$

1-(1-Butynyl)-3-(trifluoromethyl)benzene (6d). Colorless oil. IR (film) $\nu$ 2980, 1433, 1337, 1238, 1168, 1129, 1073, 801, $691 \mathrm{~cm}^{-1} ;{ }^{1} \mathrm{H}$ NMR $\left(400 \mathrm{MHz}, \mathrm{CDCl}_{3}\right) \quad \delta 1.24(\mathrm{t}, J=7.0 \mathrm{~Hz}, 3 \mathrm{H})$, $2.42(\mathrm{q}, J=7.0 \mathrm{~Hz}, 2 \mathrm{H}), 7.36-7.42(\mathrm{~m}, 1 \mathrm{H}), 7.48-7.57(\mathrm{~m}, 2 \mathrm{H}), 7.65(\mathrm{~s}, 1 \mathrm{H}) ;{ }^{13} \mathrm{C} \mathrm{NMR}(100 \mathrm{MHz}$, $\left.\mathrm{CDCl}_{3}\right) \quad \delta 13.0(\mathrm{~s}) ; 13.7(\mathrm{~s}), 78.6(\mathrm{~s}), 93.5(\mathrm{~s}), 124.1(\mathrm{q}), 125(\mathrm{~s}), 128.4(\mathrm{q}), 128.6(\mathrm{~s}), 130.7(\mathrm{q}), 134.6$ (q), 135.2 (q); EI-MS m/z (rel intensity) 198 (M+•, 79 ), 197 (68), 193 (10), 183 (52), 177 (87), 143 (18), 133 (13), 129 (50), 128 (53), 127 (12); HRMS calcd for $\mathrm{C}_{11} \mathrm{H}_{3} \mathrm{~F}_{3}$ 198.0656, found 198.0670.

1-(1-Butynyl)-2-methoxybenzene (6e). Colorless oil. IR (film) $\nu$ 2975, 2938, 1596, 1575, 1494, 1464, 1434, 1262, 1117, 1049, 1027, $752 \mathrm{~cm}^{-1} ;{ }^{1} \mathrm{H}$ NMR $\left(500 \mathrm{MHz}, \mathrm{CDCl}_{3}\right) \quad \delta 1.26(\mathrm{t}, J=5.0$ $\mathrm{Hz}, 3 \mathrm{H}), 2.48(\mathrm{q}, J=10.0 \mathrm{~Hz}, 2 \mathrm{H}), 3.87(\mathrm{~s}, 3 \mathrm{H}), 6.84-6.89(\mathrm{~m}, 2 \mathrm{H}), 7.23(\mathrm{t}, J=10.0 \mathrm{~Hz}, 1 \mathrm{H})$, 7.37-7.39 (m, 1H); ${ }^{13} \mathrm{C}$ NMR (125 MHz, $\left.\mathrm{CDCl}_{3}\right) \quad \delta 13.5$ (s), 13.9 (s), 55.8 (s), 75.9 (s), 95.9 (s), 110.5 (s), 113.1 (s), 120.4 (s), 128.9 (s), 133.7 (s), 159.8 (s); EI-MS m/z (rel intensity) 161 (12), 160 $\left(\mathrm{M}^{+\bullet}, 100\right), 146$ (20), 145 (50), 144 (10), 131 (35), 128 (10), 127 (16), 118 (10), 117 (31), 116 (15), 115 (64), 91 (34), 89 (11), 77 (15), 63 (14), 51 (13), 39 (13); Anal calcd for $\mathrm{C}_{11} \mathrm{H}_{12} \mathrm{O} \mathrm{C}, 82.46$; $\mathrm{H}$,

\footnotetext{
${ }^{6}$ Bock, H.; Rittmeyer, P.; Stein, U. Chem.Ber. 1986, 119, 3766-3781.
} 
7.55; Found $\mathrm{C}, 82.38 ; \mathrm{H}, 7.57 .{ }^{1} \mathrm{H}$ NMR spectrum in agreement with data published in literature. ${ }^{7}$

1-Ethyl-4-(1-pentynyl)benzene (6f). Colorless oil. IR (film) $\nu$ 2965, 2933, 2873, 1511, 1458, 833, $549 \mathrm{~cm}^{-1} ;{ }^{1} \mathrm{H}$ NMR $\left(400 \mathrm{MHz}, \mathrm{CDCl}_{3}\right) \quad \delta 1.04(\mathrm{t}, J=7.0 \mathrm{~Hz}, 2 \mathrm{H}), 1.21(\mathrm{t}, J=7.0 \mathrm{~Hz}, 3 \mathrm{H})$, 1.62 (sextet, $J=7.0 \mathrm{~Hz}, 2 \mathrm{H}), 2.38(\mathrm{t}, J=7.0 \mathrm{~Hz}, 2 \mathrm{H}), 2.62(\mathrm{q}, J=7.0 \mathrm{~Hz}, 2 \mathrm{H}), 7.08-7.12(\mathrm{~m}$, 2H), 7.29-7.34 (m, 2H); $\left.{ }^{13} \mathrm{C} \mathrm{NMR} \mathrm{(100} \mathrm{MHz,} \mathrm{CDCl}_{3}\right) \quad \delta 13.5$ (s), 15.3 (s), $21.4(\mathrm{~s}), 22.3(\mathrm{~s}), 28.8$ (s), 80.7 (s), $89.4(\mathrm{~s}), 121.2$ (s), 127.7 (s), 131.5 (s), 143.7 (s); EI-MS m/z (rel intensity) 173 (8), 172 $\left(\mathrm{M}^{+} \bullet, 56\right), 157$ (21), 144 (29), 143 (100), 142 (13), 141 (13), 129 (44), 128 (59), 127 (14), 115 (21), 77 (9); HRMS calcd for $\mathrm{C}_{13} \mathrm{H}_{16}$ 172.1252, found 172.1247.

3-Heptynylphenylsulfone (6g). Colorless crystals. IR (film) $\nu$ 3063, 2966, 2936, 1448, 1317, 1155, 1087, 566, $536 \mathrm{~cm}^{-1}$; ${ }^{1} \mathrm{H}$ NMR $\left(200 \mathrm{MHz}, \mathrm{CDCl}_{3}\right) \quad \delta 0.89(\mathrm{t}, J=16.6 \mathrm{~Hz}, 3 \mathrm{H}), 1.39$ (sextet, $J=7.0 \mathrm{~Hz}, 2 \mathrm{H}), 1.98(\mathrm{tt}, J=7.2$ and $2.4 \mathrm{~Hz}, 2 \mathrm{H}), 2.52-2.64(\mathrm{~m}, 2 \mathrm{H}), 3.20-3.32(\mathrm{~m}, 2 \mathrm{H}), 7.50-7.72$ $(\mathrm{m}, 3 \mathrm{H}), 7.86-7.96(\mathrm{~m}, 2 \mathrm{H}) ;{ }^{13} \mathrm{C} \mathrm{NMR}\left(50 \mathrm{MHz}, \mathrm{CDCl}_{3}\right) \quad \delta 13.4(\mathrm{~s}), 13.6(\mathrm{~s}), 20.5(\mathrm{~s}), 22.0(\mathrm{~s}), 55.2$ (s), 75.0 (s), 82.6 (s), 128.2 (s), 129.3 (s), 133.8 (s), 138.6 (s); EI-MS m/z (rel intensity) 208 (7), 143 (93), 129 (10), 125 (19), 111 (18), 94 (33), 93 (14), 79 (100), 77 (44), 67 (34), 55 (27); HRMS calcd for $\mathrm{C}_{13} \mathrm{H}_{16} \mathrm{O}_{2} \mathrm{~S} 236.0871$, found 236.0862 .

\section{NMR and ESI Spectra}

7 Aitken, R. A.; Burns, G. J. Chem. Soc. Perkin. Trans. 1 1994, 17, 2455-2460. 


\section{Compound 3c}

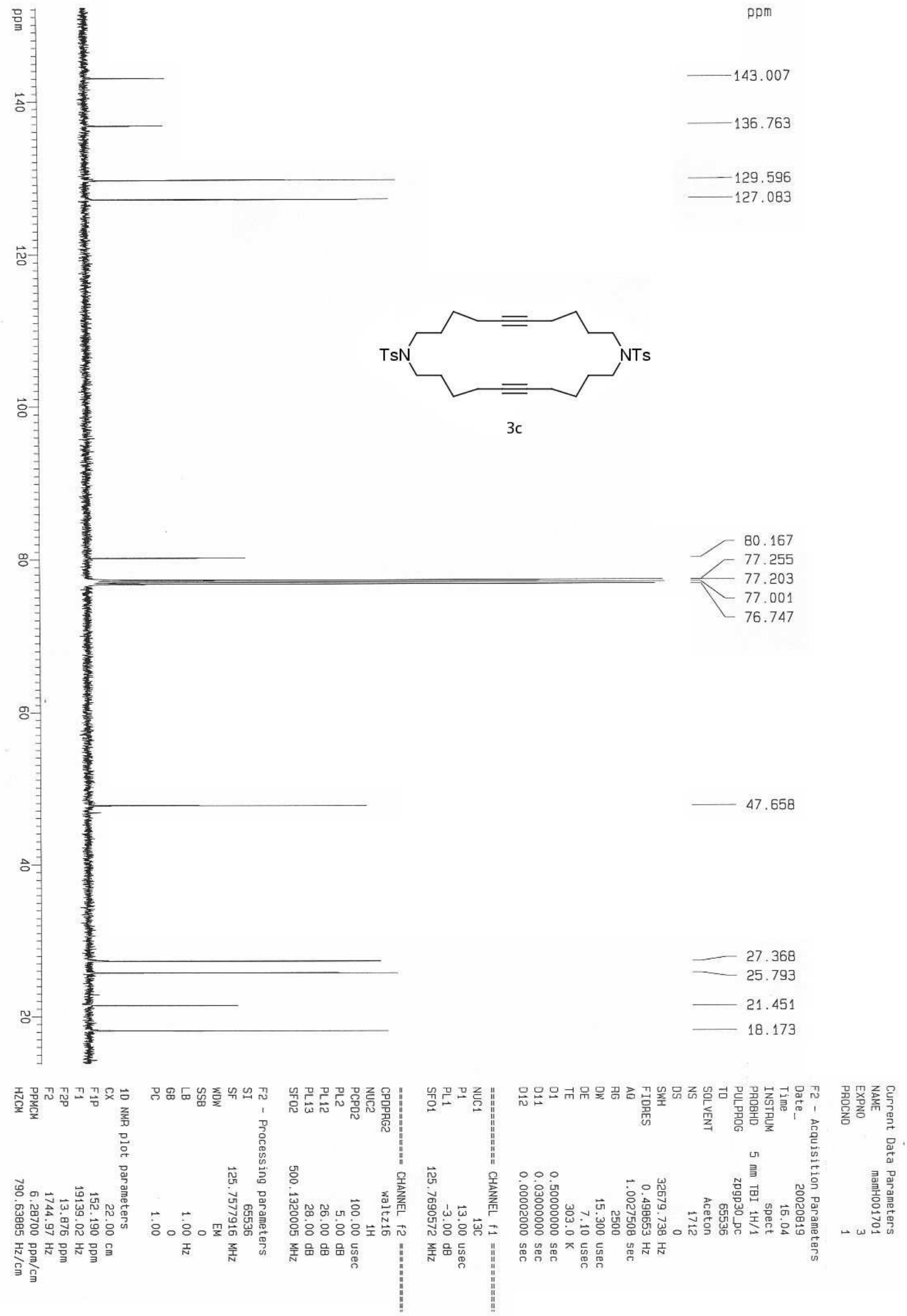




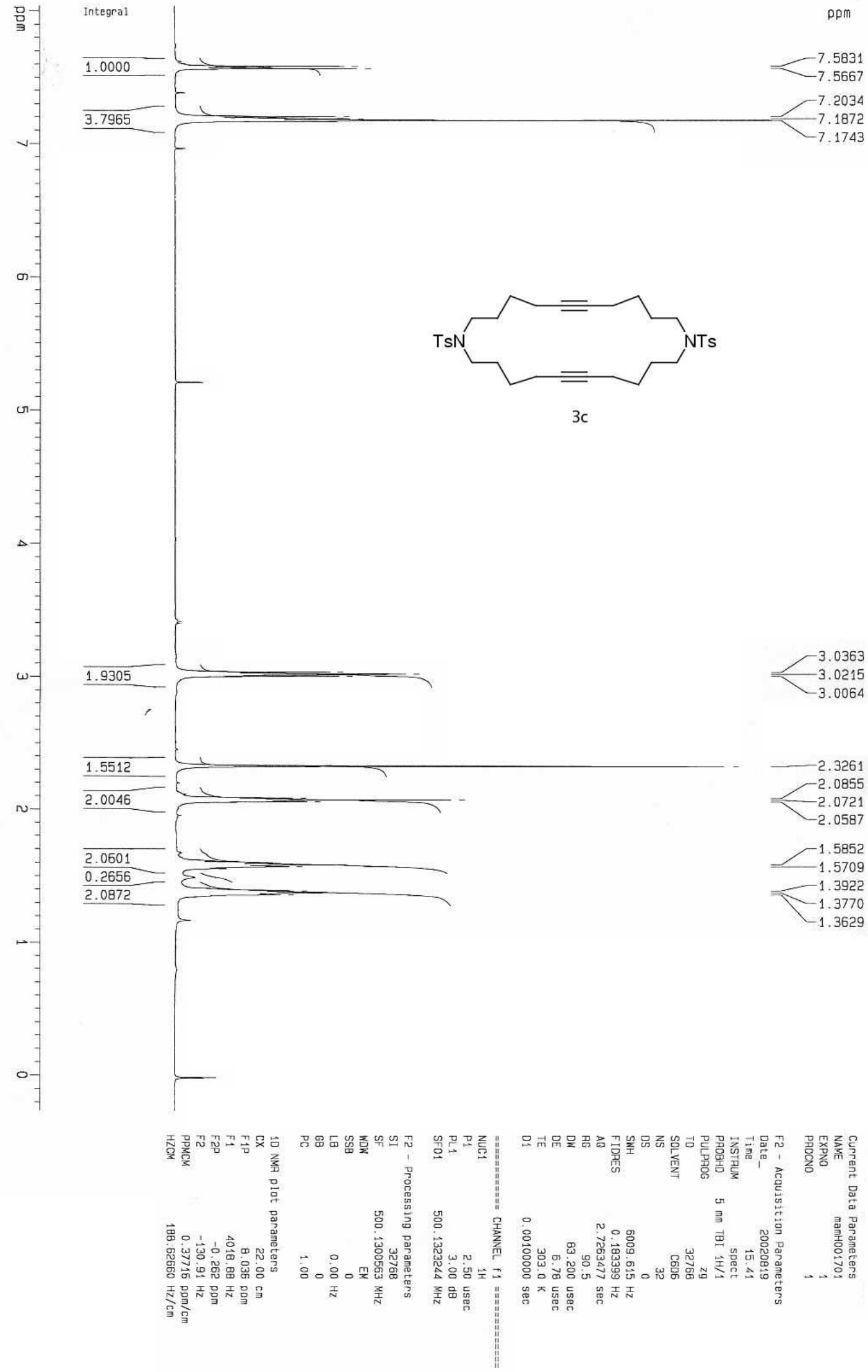




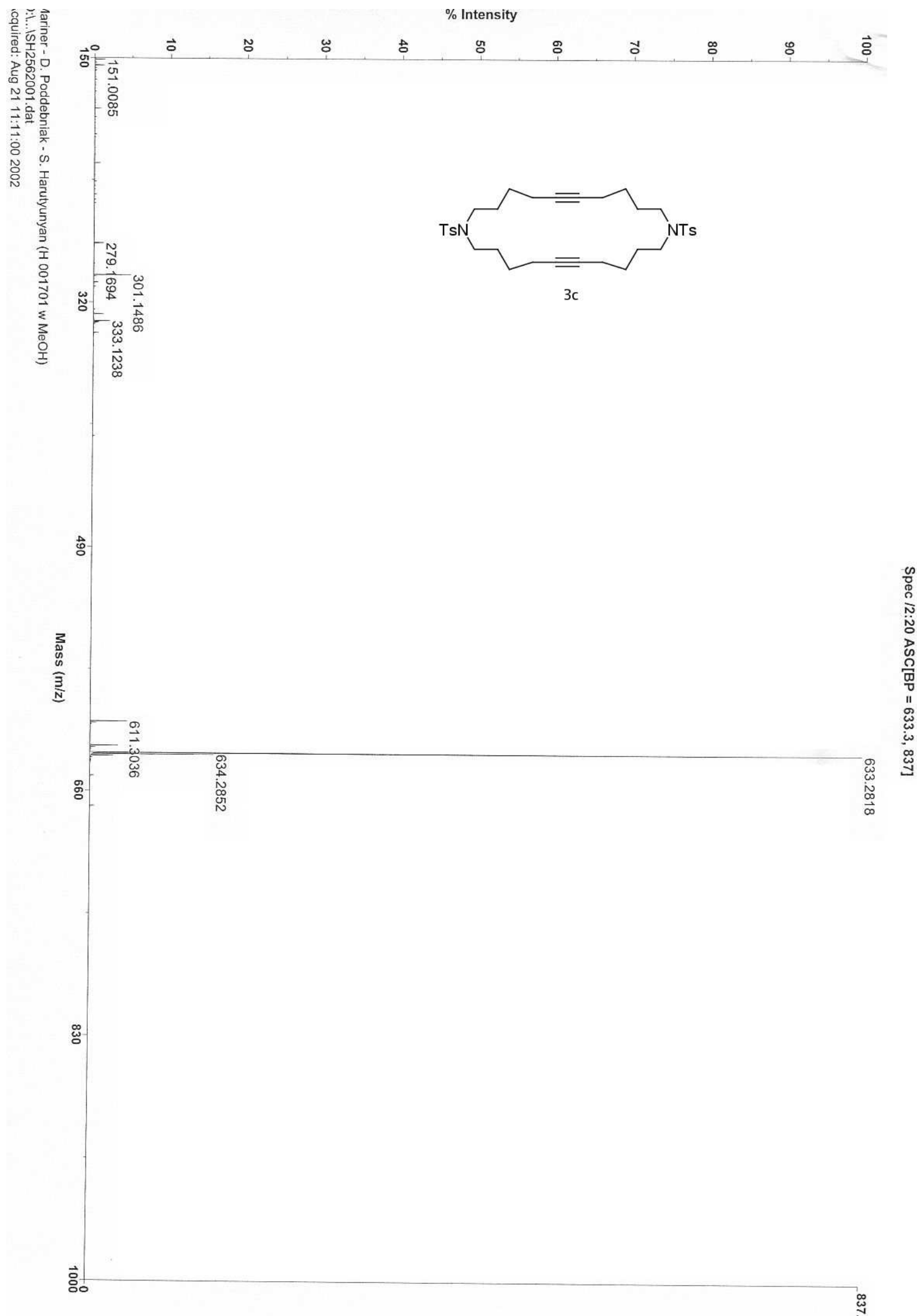




\section{Compound $5 f$}

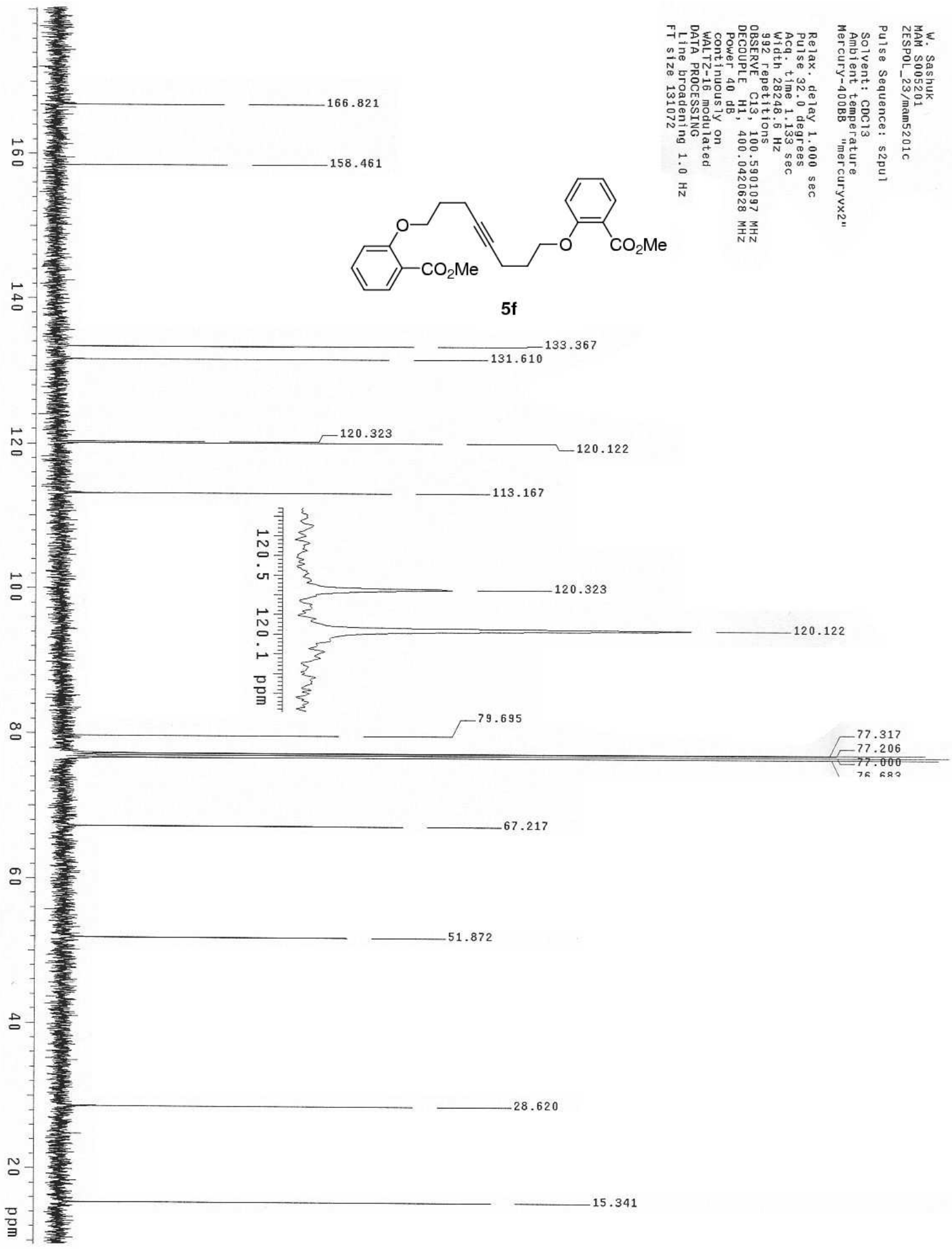




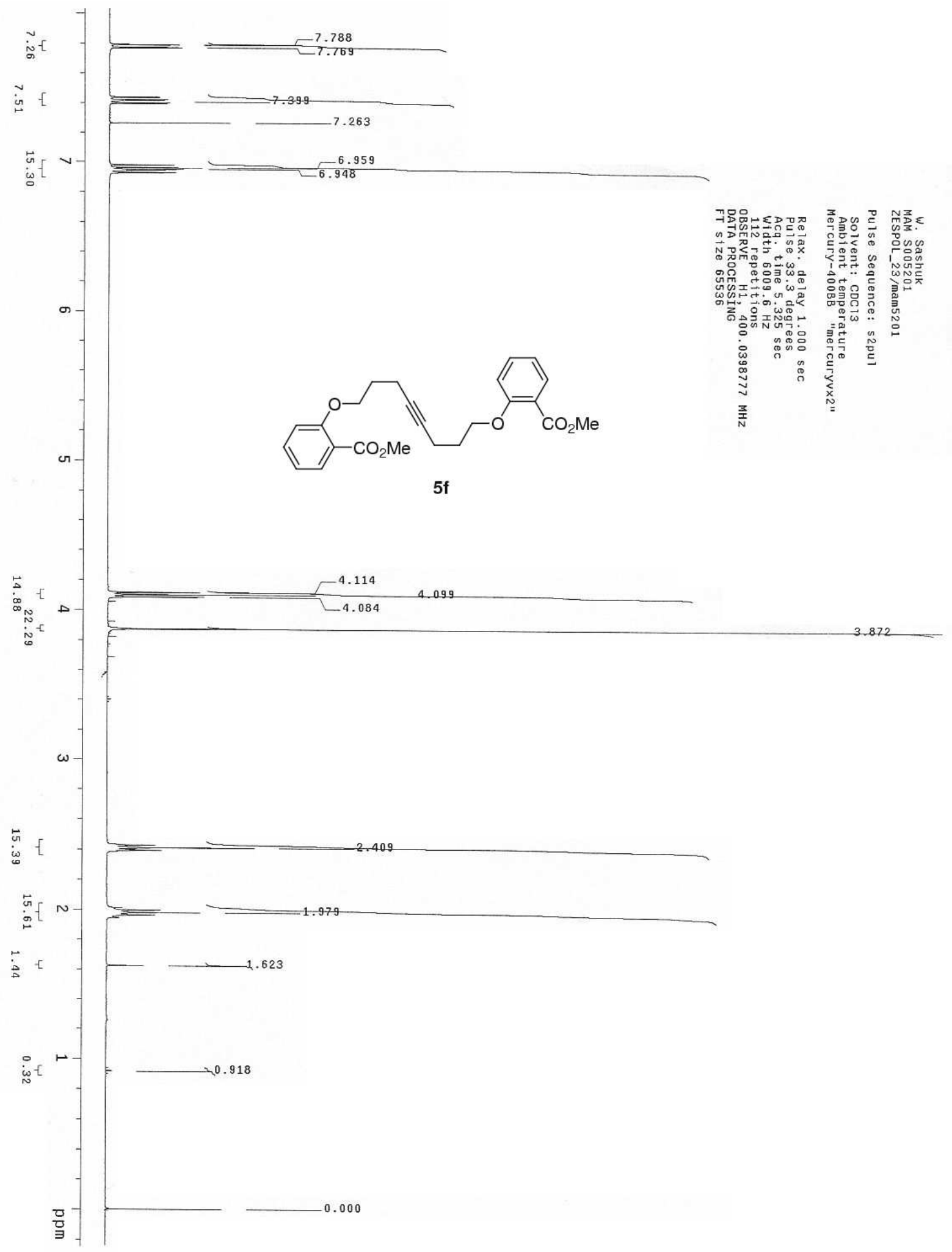




\section{Compound $5 g$}
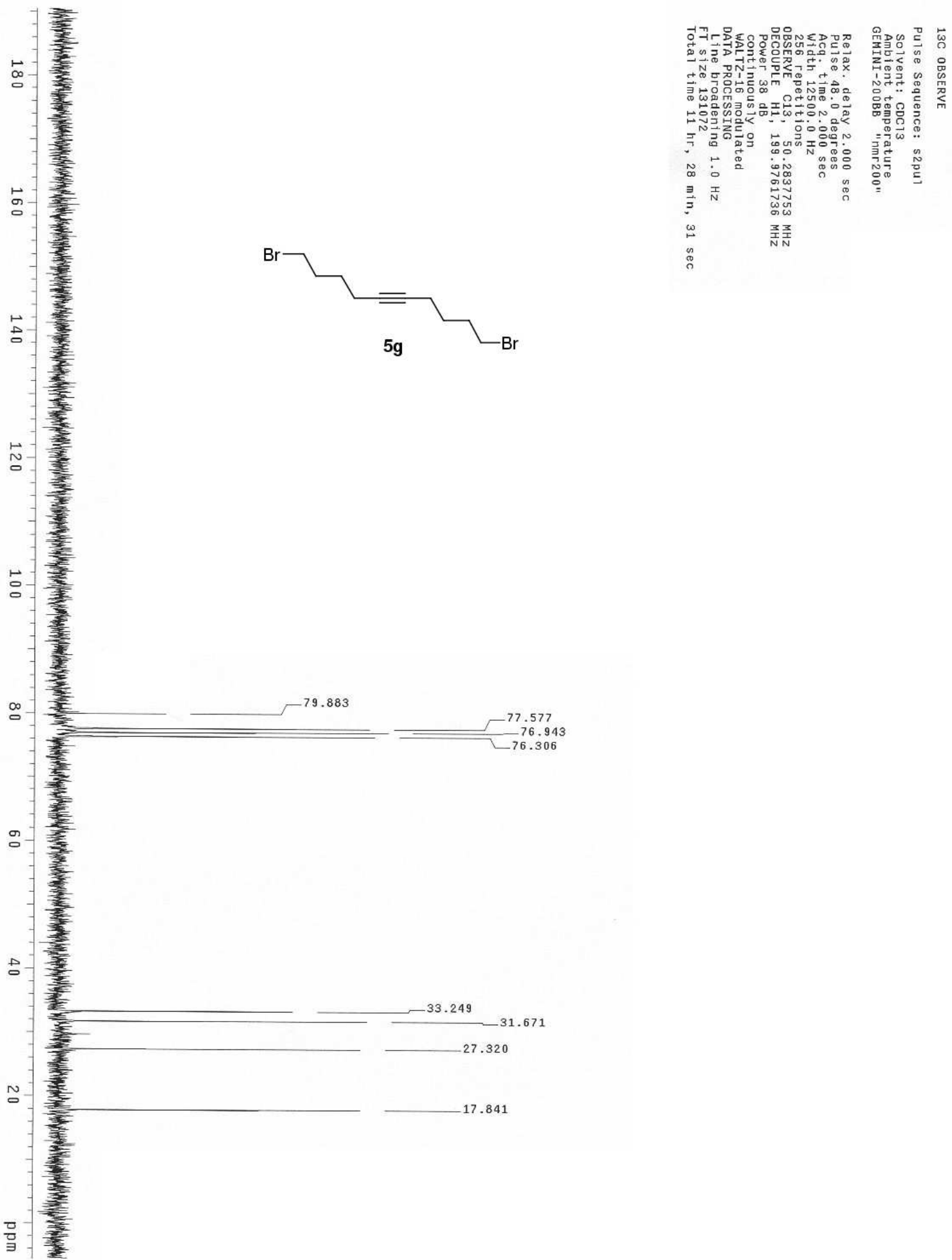

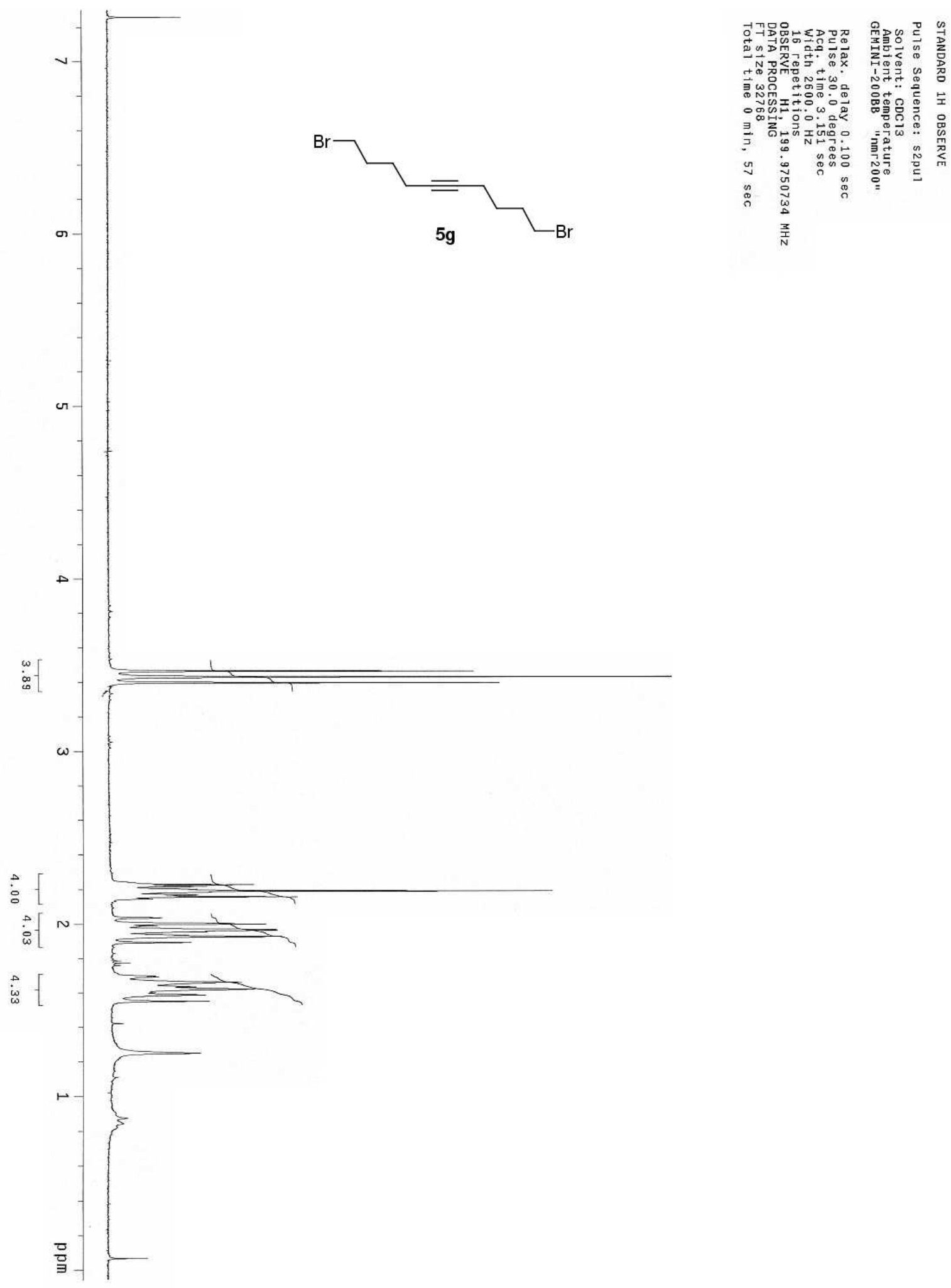


\section{Compound 6a}
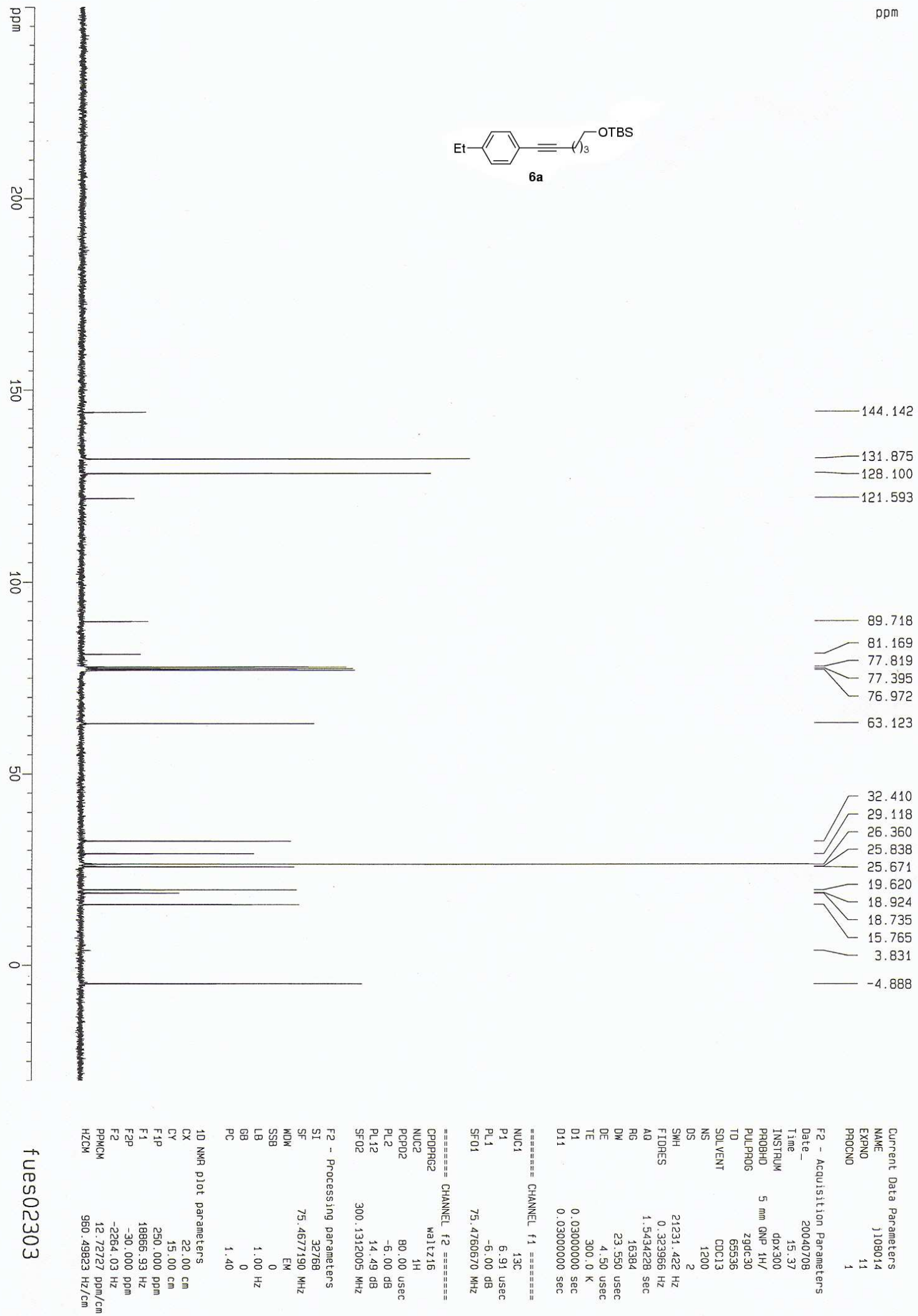


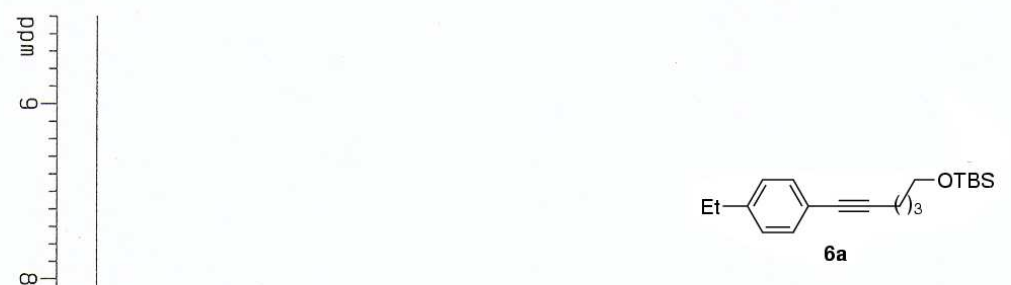

ppm

7.2601

7.2330

7.0571

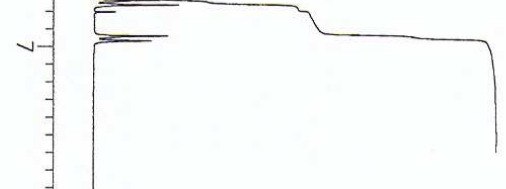




\section{Compound 6d}

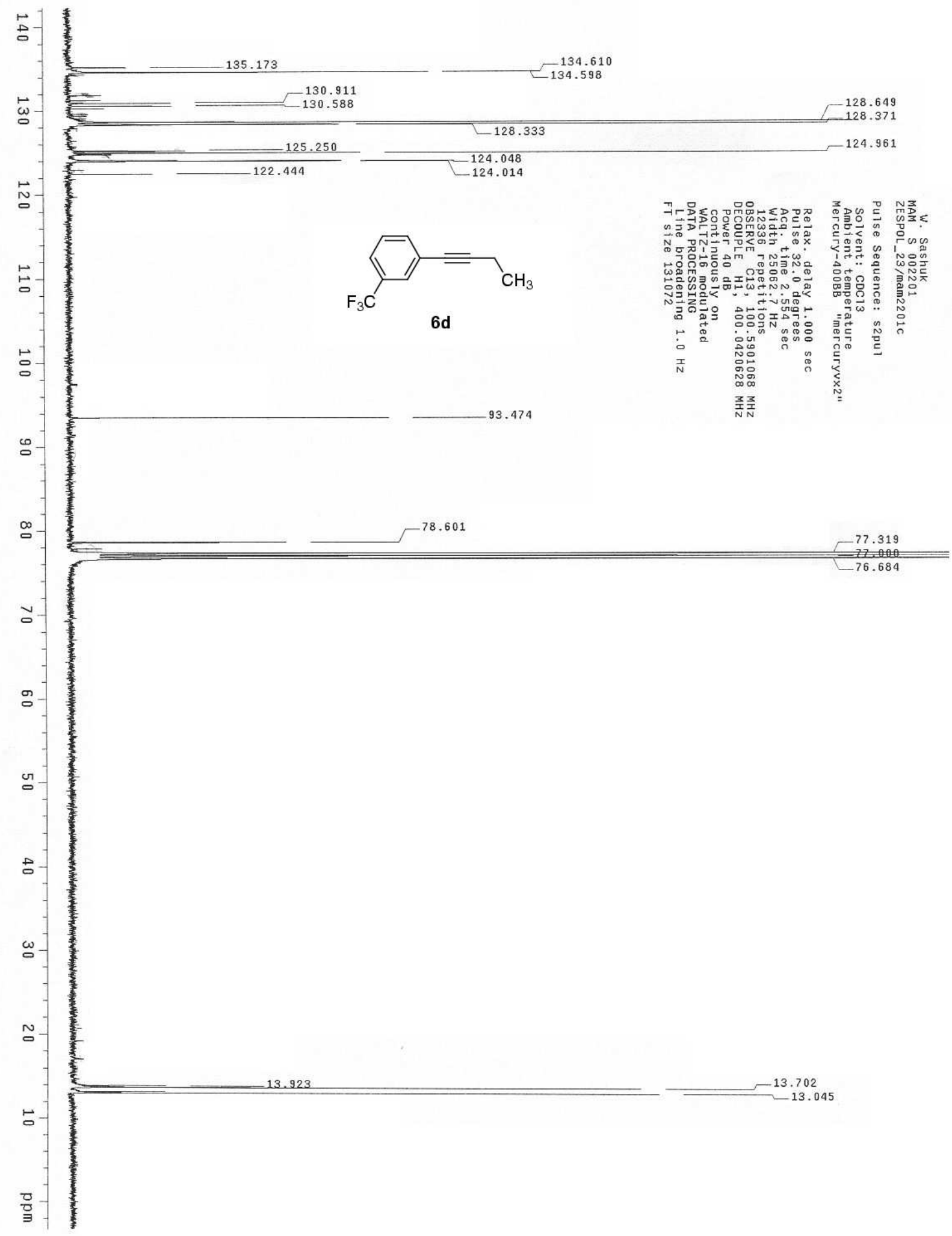




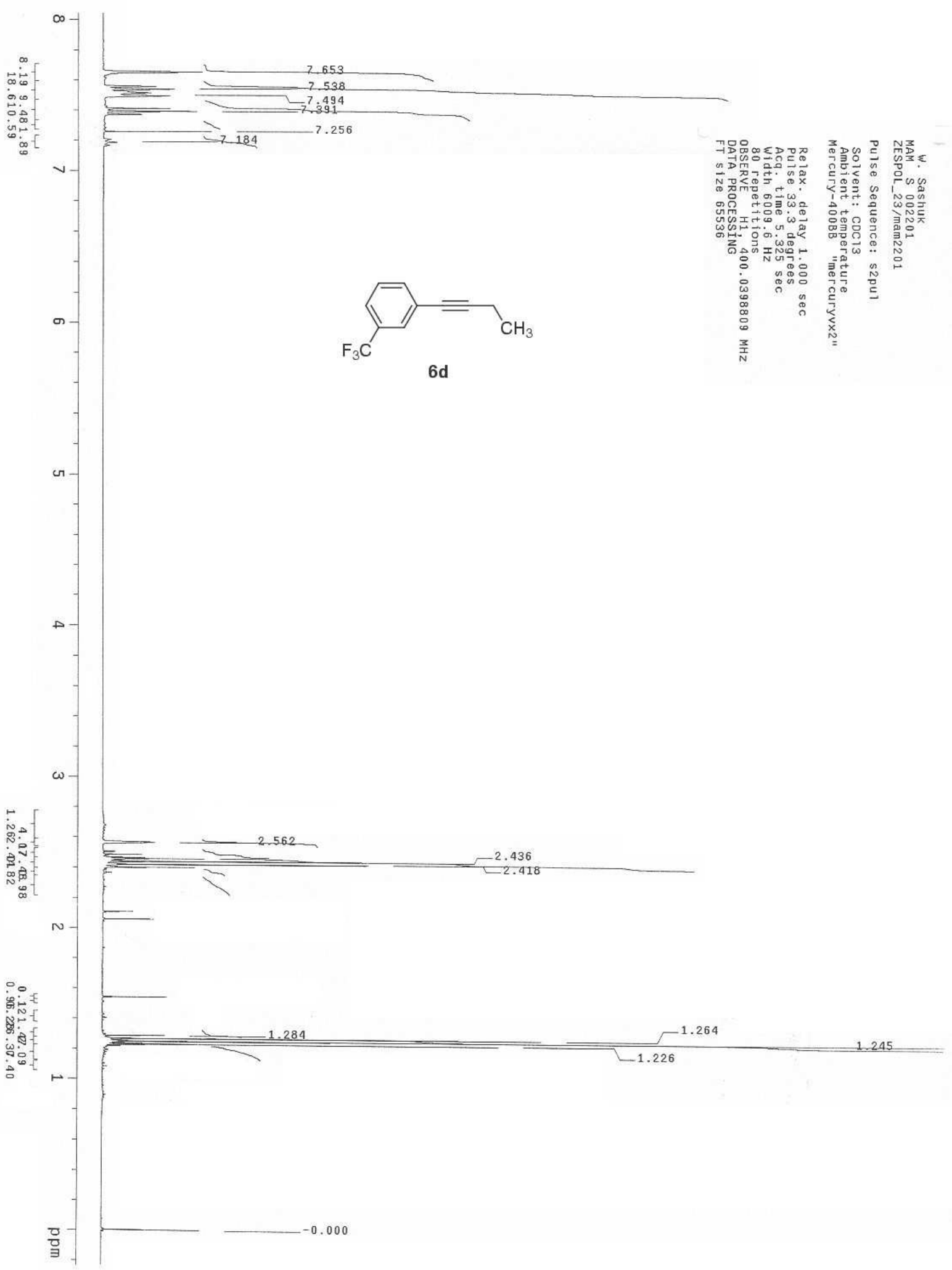




\section{Compound 6f}

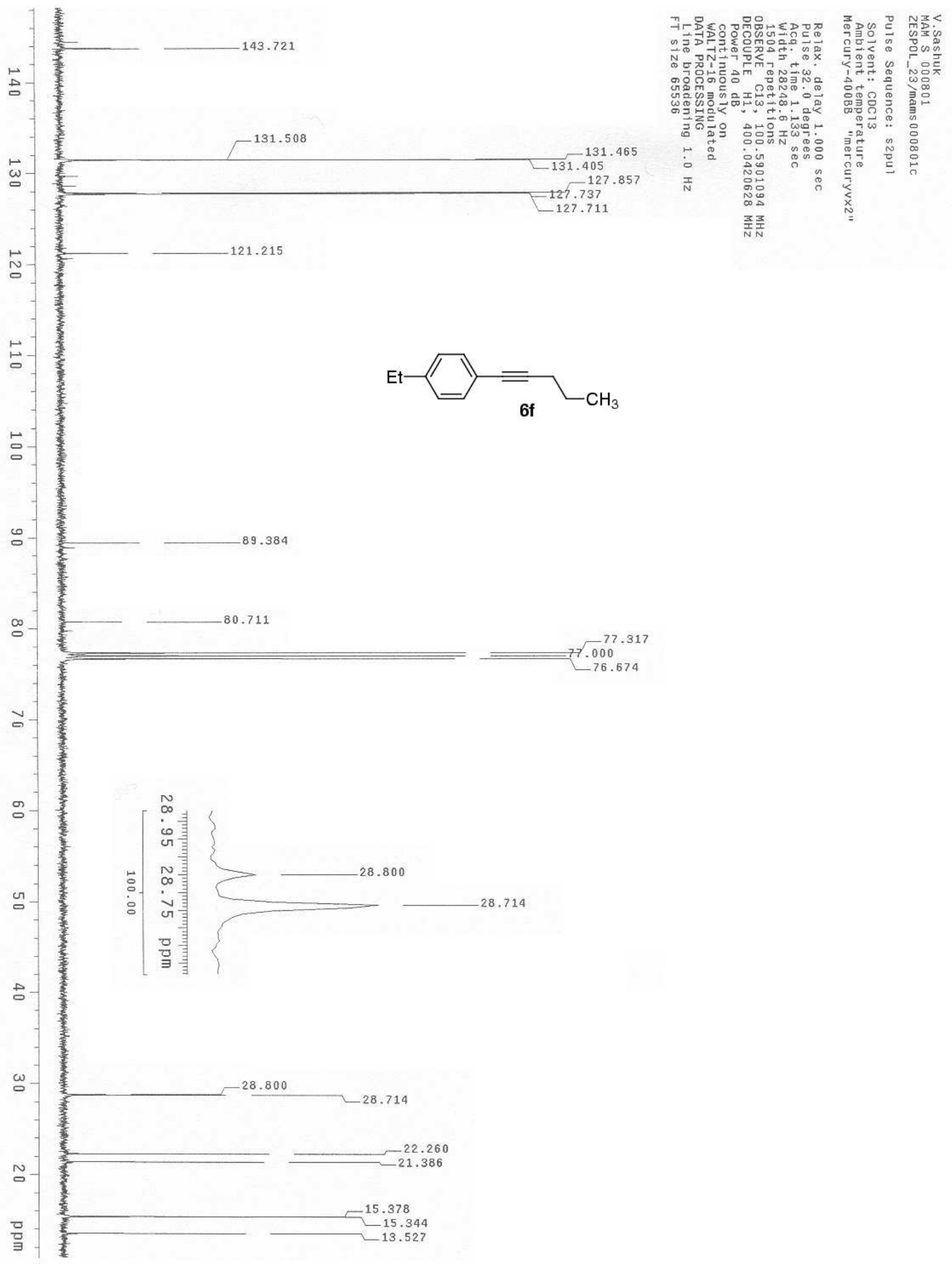




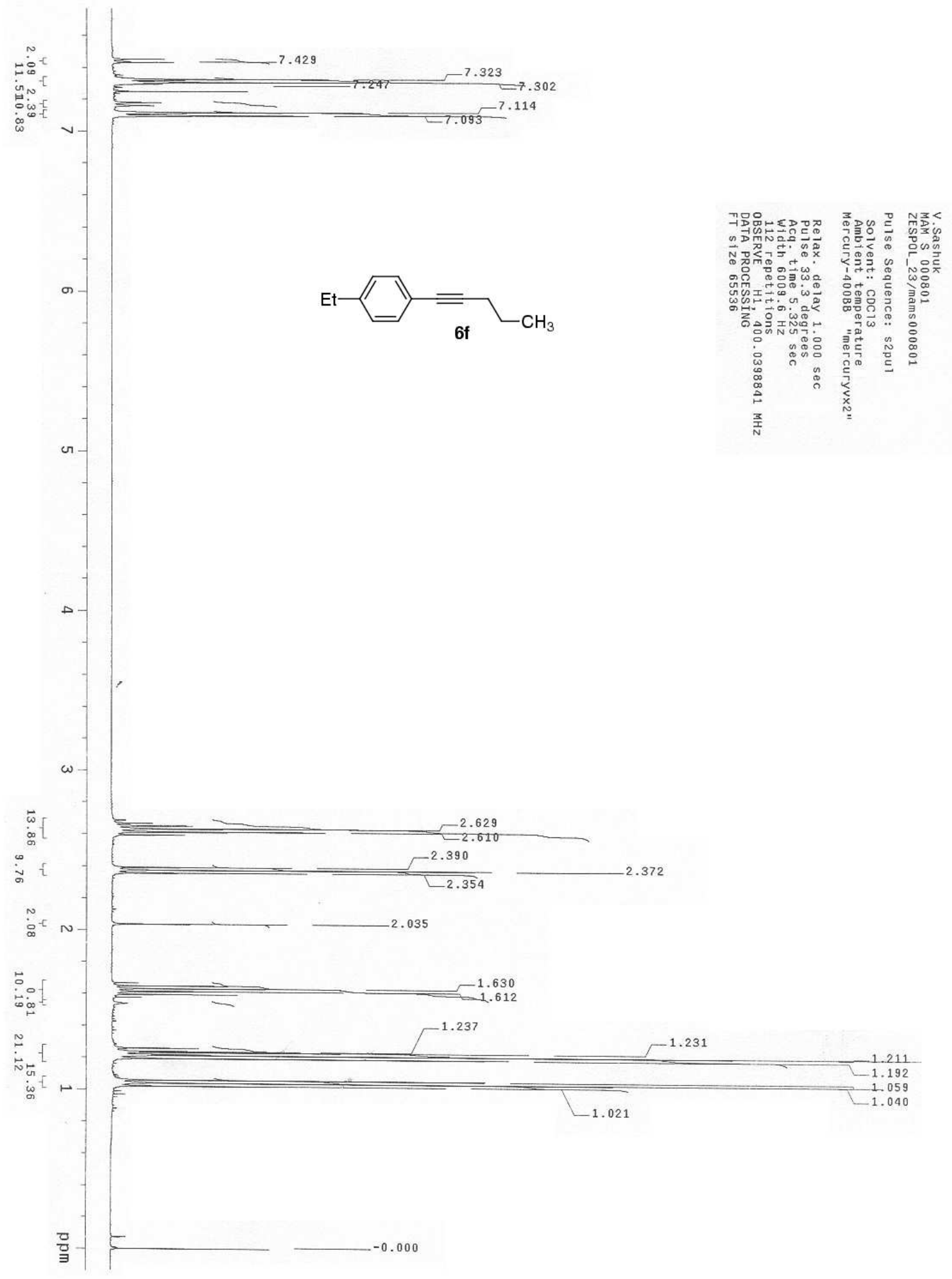




\section{Compound $6 \mathrm{~g}$}

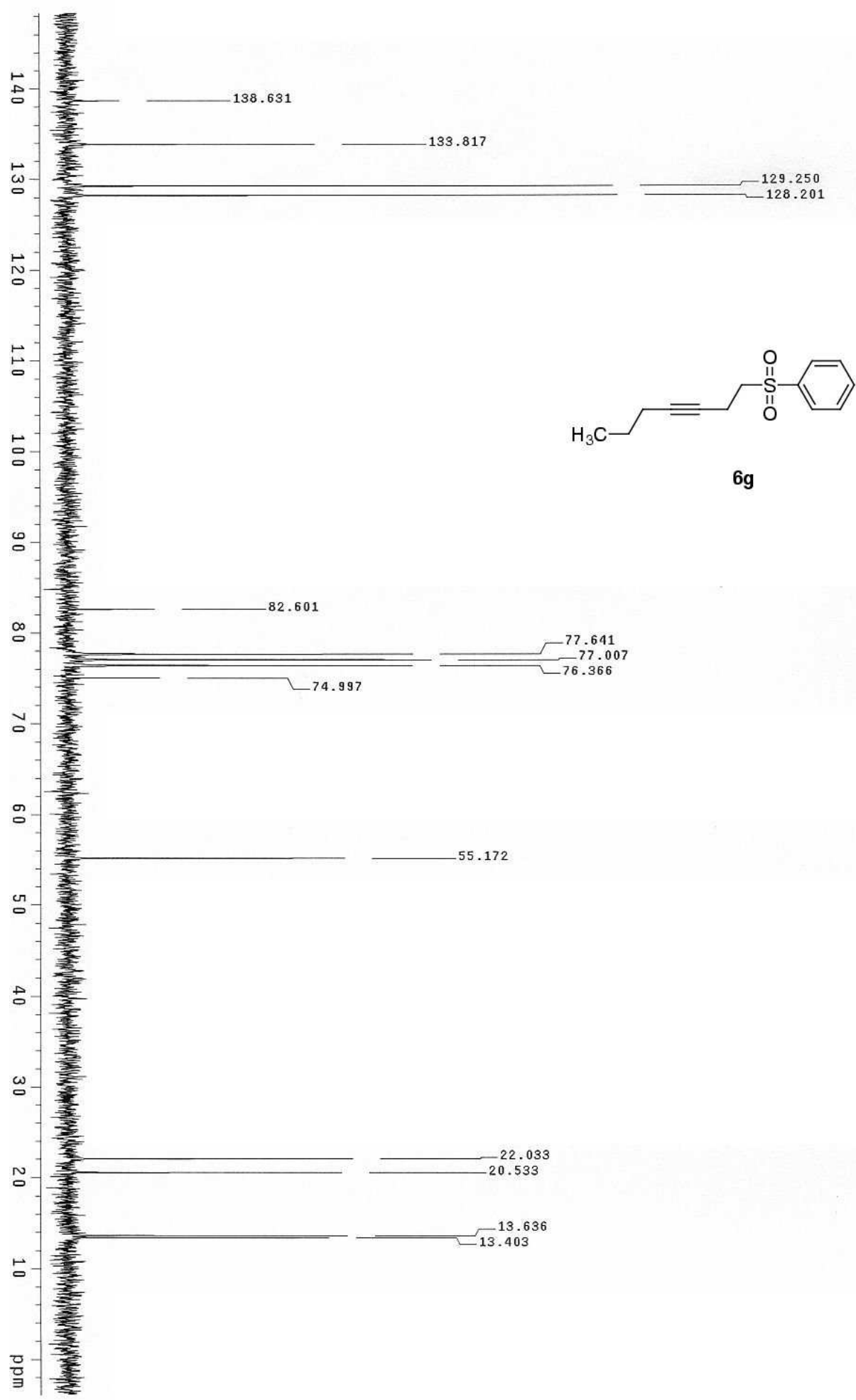




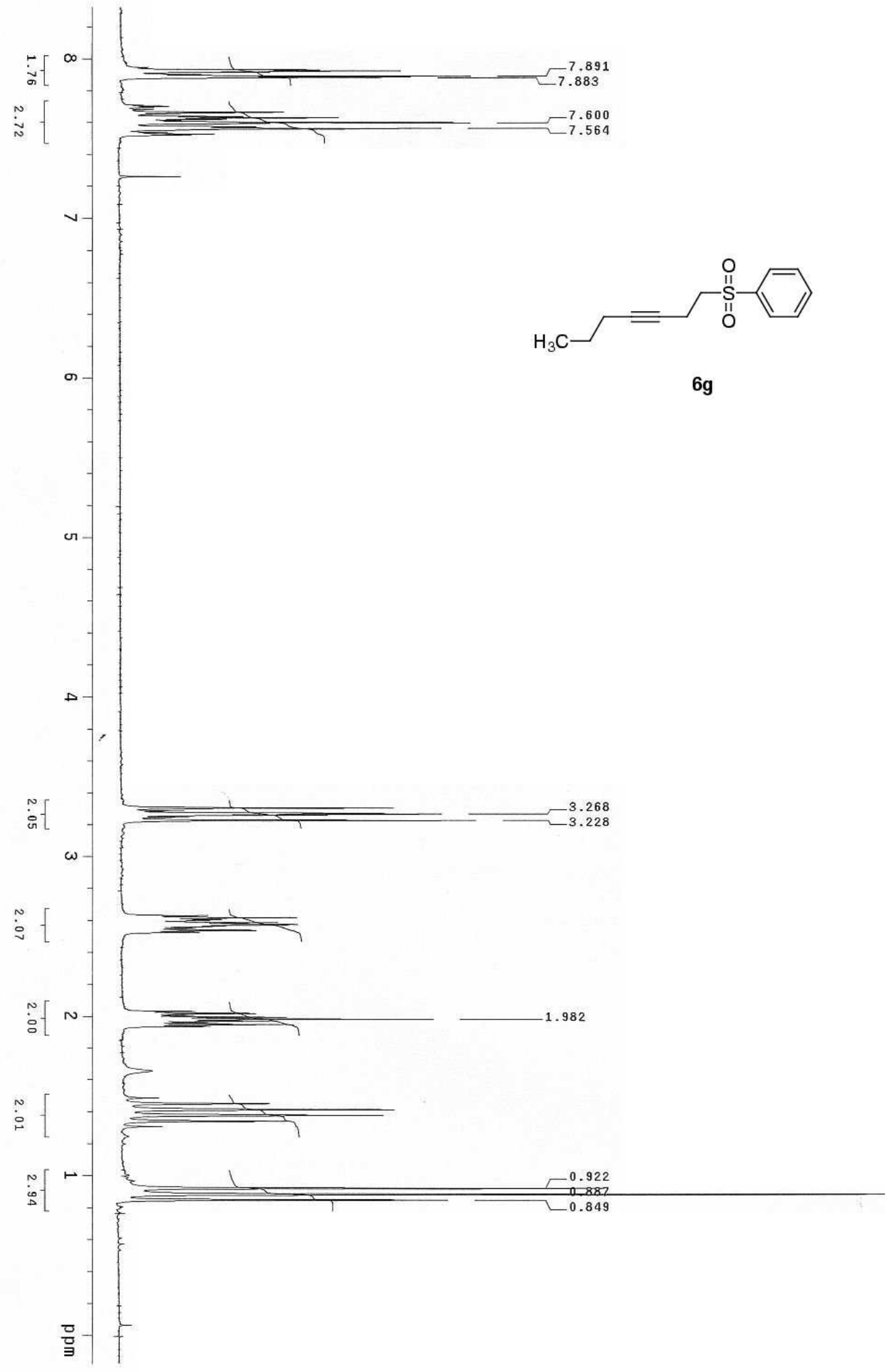

